\title{
Immunohistochemical expression of $\beta$ - catenin, Ki67, CD3 and CD18 in canine colorectal adenomas and adenocarcinomas
}

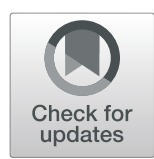

Kristin M. V. Herstad ${ }^{1 *}$ (D) Gjermund Gunnes ${ }^{2}$, Runa Rørtveit ${ }^{2}, \varnothing y$ vor Kolbjørnsen ${ }^{3}$, Linh $\operatorname{Tran}^{3}$ and Ellen Skancke ${ }^{1}$

\begin{abstract}
Background: Inflammation is believed to influence human colorectal carcinogenesis and may have an impact on prognosis and survival. The mucosal immunophenotype in dogs with colorectal cancer is poorly described.

The aim of this study was to investigate whether the density, distribution and grade of tumor-infiltrating immune cells (TIIs) are different in normal colonic tissue vs benign stages (adenomas) and malignant stages (adenocarcinomas) of canine colorectal carcinogenesis, and thus, whether they can be considered as prognostic factors in dogs. This retrospective case-control study was performed on formalin-fixed, paraffin-embedded tissue samples from dogs with histologically confirmed colorectal adenoma $(n=18)$ and adenocarcinoma $(n=13)$ collected from archived samples. The samples had been collected by colonoscopy, surgery or during postmortem examination. Healthy colonic tissue obtained post mortem from dogs euthanized for reasons not involving the gastrointestinal tract served as control tissue $(n=9)$.

Results: The tumor samples had significantly lower numbers of CD3+ T-cells in the epithelium compared to controls (adenocarcinoma vs control, Kruskal-Wallis test, $p=0.0004$, and adenoma vs control, $p=0.002$ ). Adenomas had a significantly lower number of CD18+ cells in the lamina propria, compared to control samples (Kruskal-Wallis test, $p=$ 0.008). Colonic samples from control dogs had uniform staining of $\beta$-catenin along the cell membrane of epithelial cells. Compared to normal colonic cells, the expression levels of cytoplasmic $\beta$-catenin were significantly higher in adenomas and adenocarcinomas (adenoma vs control Kruskal-Wallis test, $p=0.004$, and adenocarcinoma vs control, $p=0.002$ ). None of the control samples showed positive staining of $\beta$-catenin in the nucleus of colonic cells. In contrast, adenocarcinomas and adenomas showed moderate to strong staining of the cell nucleus. The nuclear $\beta$-catenin expression (signal strength and distribution) was significantly higher in adenomas compared to adenocarcinomas (Kruskal-Wallis test, $p<0.05$ ).

Conclusions: $\beta$-catenin and Ki67 were not useful markers for demonstrating tumor progression from adenomas to adenocarcinomas. The lower presence of CD18 and CD3+ cells in colorectal tumors compared to controls indicates a reduced presence of histiocytes and T-cells, which may have implications for the pathogenesis and progression of colorectal cancer in dogs.
\end{abstract}

Keywords: Canine, Colorectal adenoma and adenocarcinoma, Markers for tumor progression, Tumor-infiltrating immune cells

\footnotetext{
* Correspondence: kristin.herstad@nmbu.no

${ }^{1}$ Department of Companion Animal Clinical Sciences, Faculty of Veterinary Medicine, Norwegian University of Life Sciences (NMBU), Oslo, Norway

Full list of author information is available at the end of the article
} 


\section{Background}

Colorectal cancer $(\mathrm{CRC})$ is one of the most common types of cancer in humans [1]. In contrast to humans, $\mathrm{CRC}$ is rarely diagnosed in dogs, albeit more frequently than in other mammals [2, 3]. The disease is associated with serious clinical signs and has a poor prognosis due to local recurrence and metastases in humans $[4,5]$ and dogs [6-8]. Sporadic CRC in humans is believed to originate from adenomas through a process of multiple genetic and molecular events [9]. Studies indicate that a similar process occurs in canine colorectal carcinogenesis [10-14].

Colonic stem cells are located at the bottom of the colonic crypt where they proliferate and migrate towards the top, resulting in mature cells without the capacity to divide. This proliferation process is tightly regulated through the wingless-related integration site (Wnt) signalling pathway and involves the protein $\beta$-catenin [15]. Mutation of $\beta$-catenin is a key factor in CRC tumorigenesis, as described in humans [15] and dogs [10, 16, 17]. A failure in this mechanism results in colonic cells with increased proliferation capacity. Cellular proliferation in tumorigenesis may be evaluated by Ki67 [18]. The Ki67protein is only present during active phases of the cell cycle; thus, expression of this antigen indicates cellgrowth [19].

Inflammatory cells have been proposed to play a role in colorectal carcinogenesis in humans [20, 21], and chronic inflammation such as ulcerative colitis may progress to cancer [22]. In humans, inflammatory cells infiltrating colorectal adenoma and adenocarcinoma may influence the tumour's capability to proliferate and metastasize [23]. The presence of tumor-infiltrating CD3+ T-lymphocytes in human CRC correlates with prognosis, as patients with high tumor infiltration have longer survival times than those with poorly infiltrated tumors [24]. CD18 is a panleucocyte marker, often used to characterize histiocytic cells, including monocytes and macrophages [25]. These macrophages, referred to as tumor-associated macrophages (TAMs), contribute to metastases in human colorectal carcinogenesis [26].

In Japan, miniature dachshunds seem to be predisposed to inflammatory polyps which may develop into adenoma and adenocarcinoma [17, 27, 28]. However, in these studies, TIIs were only described in inflammatory polyps and not in adenomas and adenocarcinomas [17, 28]. To the best of the authors' knowledge, tumorinfiltrating immune-cells (TIIs) in canine CRC have not previously been characterized. In this work, we characterized and quantified the infiltration of immune cells in canine colorectal adenoma, adenocarcinoma, and normal colonic tissue using the antigens CD18 and CD3 labelling histiocytes and T-cells, respectively. Evaluation of tumor progression was performed using the antigens $\beta$ catenin and Ki67.

\section{Results}

No significant difference in breed, age and gender were noted among dogs with colorectal adenoma, adenocarcinoma and control dogs (Kruskal-Wallis test, $p>0.1$ ).

For the dogs with tumors, the following breeds were represented with $\geq 3$ individuals: German Shepherd ( $n=$ $3)$ and English Setter $(n=3)$. The control dogs consisted of various breeds of which each was represented only once in the study material.

Information regarding tumor location was available in 22 dogs. The tumors were located in the rectum in 18 dogs, and in the colon in four. The localization of the remaining nine tumors was not specified (Table 1 ).

The number of CD3+ cells in the epithelium was significantly lower in adenomas $(n=18)$ and adenocarcinomas $(n=13)$ compared to control samples $(n=6)$ [Kruskal-Wallis test, adenomas vs controls, median 0 (IQR, 0-0) vs $1.5(1-2), p=0.002$ and adenocarcinomas vs controls, $0(0-0)$ vs $1.5(1-2), p=0.0004]$. No difference was detected in lamina propria CD3+ cell-numbers between tumor samples and control samples (KruskalWallis test, $p>0.1$, Fig. $1 \mathrm{a}$ and $\mathrm{b})$.

The adenoma samples $(n=18)$ had a significantly lower expression of CD18 positive cells in the lamina propria, compared to control samples $(n=8)$ [KruskalWallis test, median 1 (IQR, 0-1.5) vs 2.25 (1.63-3), $p=$ 0.008]. Adenocarcinomas $(n=12)$ had a significantly higher presence of CD18 positive cells when compared with adenomas $(n=18)$ [Kruskal-Wallis test, $1.75(1-2)$ vs 1 (0-1.5), $p=0.05$, Fig. $1 \mathrm{c}$ and d]. Although control samples had higher CD18 expression than tumor samples, this difference was not significant when comparing adenocarcinoma samples with control samples [KruskalWallis test, $1.75(1-2)$ vs $2.3(1.7-3) p>0.1]$.

The Ki67 positive cells showed a strong signal in adenomas $(n=14)$ and adenocarcinomas $(n=13)$, but no significant difference was detected in expression levels between these two tumor stages (Wilcoxon $p>0.05$, Fig. 1e and f). None of the control samples expressed Ki67; thus, the IHC scoring of Ki67 was only reported in tumor samples.

Colonic samples from control dogs had uniform staining of $\beta$-catenin along the cell membrane of epithelial cells. Compared to normal colonic tissue, the expression levels of cytoplasmic $\beta$-catenin were significantly higher in adenomas and adenocarcinomas (Kruskal-Wallis test, adenomas vs controls, $p=0.004$ and adenocarcinomas vs controls, $p=0.002$, Table 2 and Fig. $1 \mathrm{~g}$ and $\mathrm{h}$ ).

There was a significant difference in $\beta$-catenin expression (signal strength and distribution) when comparing tumor samples with controls (Kruskal-Wallis test, $p<$ 0.05 , Table 2). None of the control samples showed any positive staining of $\beta$-catenin in the nucleus of colonic cells. In contrast, $16 / 31$ of the tumor samples had 
Table 1 Overview of dogs and samples

\begin{tabular}{|c|c|c|c|c|c|c|c|}
\hline Dog no. & Breed & Gender & Age $(y)$ & Diagnosis $^{a}$ & $\begin{array}{l}\text { Tumor location } \\
\text { (C/R) }\end{array}$ & Method of sampling & Treatment \\
\hline 1 & German shepherd & $M$ & 9 & Adenocarcinoma & $\mathrm{R}$ & Surgery & Surgery \\
\hline 2 & Irish Setter & $\mathrm{F}$ & 10 & Adenocarcinoma & $\mathrm{R}$ & Colonoscopy & Surgery \\
\hline 3 & Shetland sheepdog & M & 14 & Adenocarcinoma & C & Post mortem & Meloxicam \\
\hline 4 & English springer spaniel & M & 8 & Adenocarcinoma & $\mathrm{R}$ & Colonoscopy & Piroxicam \\
\hline 5 & Tibetan spaniel & $\mathrm{M} / \mathrm{N}$ & 10 & Adenocarcinoma & $\mathrm{R}$ & Postmortem & Meloxicam \\
\hline 6 & UN & M & 10 & Adenocarcinoma & UN & UN & UN \\
\hline 7 & Doberman Pinscher & M & 7 & Adenocarcinoma & UN & UN & UN \\
\hline 8 & Great Dane & $\mathrm{F} / \mathrm{N}$ & 6 & Adenocarcinoma & UN & UN & UN \\
\hline 9 & UN & $\mathrm{M} / \mathrm{N}$ & 9 & Adenocarcinoma & UN & UN & UN \\
\hline 10 & UN & $\mathrm{F}$ & 8 & Adenocarcinoma & R & UN & UN \\
\hline 11 & Longhaired Collie & M & 13 & Adenocarcinoma & $\mathrm{R}$ & UN & UN \\
\hline 12 & Bernese mountain dog & M & 5 & Adenocarcinoma & $\mathrm{R}$ & UN & UN \\
\hline 13 & Flatcoated Retriever & $\mathrm{F}$ & 9 & Adenocarcinoma & UN & UN & UN \\
\hline 14 & German Shepherd & $\mathrm{F}$ & 9 & Adenoma & UN & UN & UN \\
\hline 15 & Irish Setter & M & 6 & Adenoma & UN & UN & UN \\
\hline 16 & English Setter & M & 8 & Adenoma & UN & UN & UN \\
\hline 17 & Mixed breed & M & 10 & Adenoma & R & Surgery & UN \\
\hline 18 & German Shepherd & M & 4 & Adenoma & $\mathrm{R}$ & Surgery & Surgery \\
\hline 19 & Staffordshire Bullterrier & M & 8 & Adenoma & $\mathrm{R}$ & Surgery & Surgery \\
\hline 20 & Papillon & M & 10 & Adenoma & R & Surgery & Surgery \\
\hline 21 & Collie Shorthaired & M & 3 & Adenoma & $\mathrm{R}$ & Surgery & Surgery \\
\hline 22 & Norwegian Lundehund & M & 7 & Adenoma & $\mathrm{R}$ & Colonoscopy & Surgery \\
\hline 23 & Cocker Spaniel & $\mathrm{F}$ & 10 & Adenoma & C & Colonoscopy & no \\
\hline 24 & Golden Retriever & M & 2 & Adenoma & $\mathrm{R}$ & Surgery & Surgery \\
\hline 25 & Bichon Havanais & M & 5 & Adenoma & $\mathrm{R}$ & Colonoscopy & Surgery \\
\hline 26 & English Setter & M & 11 & Adenoma & $\mathrm{R}$ & Surgery & Surgery \\
\hline 27 & Gordon Setter & $\mathrm{F}$ & 10 & Adenoma & $\mathrm{R}$ & Surgery & Surgery \\
\hline 28 & Great Dane & M & 10 & Adenoma & C & Colonoscopy & Piroxicam \\
\hline 29 & Cocker Spaniel & M & 12 & Adenoma & C & Colonoscopy & no \\
\hline 30 & Border Collie & $\mathrm{F}$ & 12 & Adenoma & $\mathrm{R}$ & Colonoscopy & Surgery \\
\hline 31 & English Setter & $\mathrm{F}$ & 8 & Adenoma & UN & UN & UN \\
\hline 32 & West Highland White Terrier & M & 15 & Respiratory distress & NA & Post mortem & NA \\
\hline 33 & $\begin{array}{l}\text { Miniature } \\
\text { Pinscher }\end{array}$ & $\mathrm{F}$ & 12 & Lung tumor & NA & Post mortem & NA \\
\hline 34 & Staffordshire Bullterrier & $\mathrm{F}$ & 13 & General weakness & NA & Post mortem & NA \\
\hline 35 & French Bulldog & M & 3 & Intervertebral disk hernia & NA & Post mortem & NA \\
\hline 36 & Alaskan Malamute & $\mathrm{F}$ & 7 & Polyneuropathy & NA & Post mortem & NA \\
\hline 37 & French Bulldog & $\mathrm{F}$ & 3 & Degenerative disk disease & NA & Post mortem & NA \\
\hline 38 & Collie, Longhair & M & UN & Epilepsy & NA & Post mortem & NA \\
\hline 39 & Pug & M & 5 & Urolithiasis & NA & Post mortem & NA \\
\hline 40 & Chihuahua & $\mathrm{M} / \mathrm{N}$ & 3 & Multiple fractures, RTA & NA & Post mortem & NA \\
\hline
\end{tabular}

${ }^{a}$ The diagnosis was not determined for all control dogs; thus symptoms/syndromes are described in some of the cases UN Unknown 

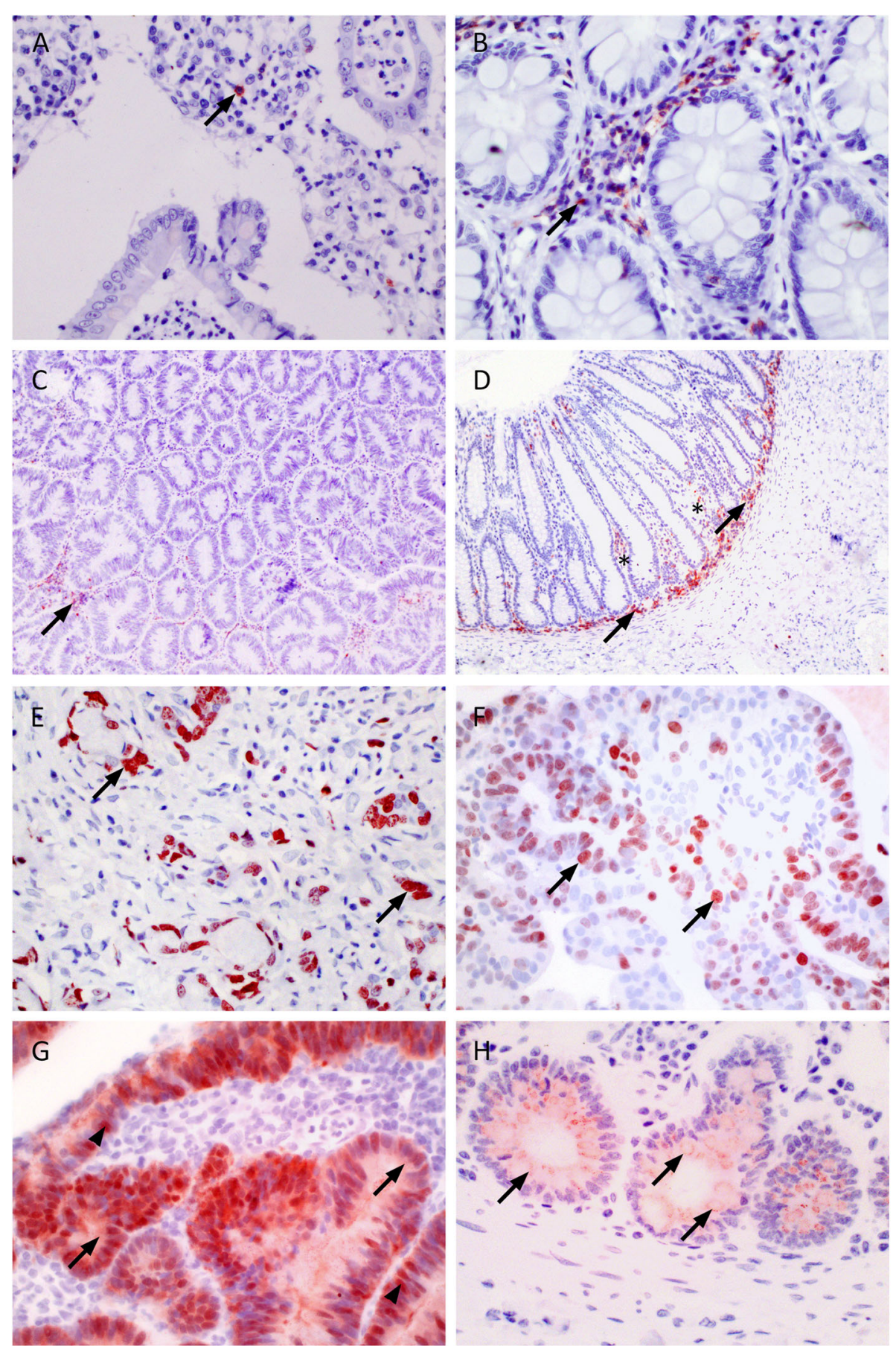

Fig. $1 \mathrm{Imm}$ unohistochemistry. a. CD3, adenocarcinoma. A small number of positive cells were found in the lamina propria (arrow). 40x. b. CD3, normal colon. Scattered cells and clusters of cells were seen in the lamina propria (arrow) and the epithelium. 40x. c. CD18, adenocarcinoma. Fewer positive cells (arrow) and weaker signal than in the control. 10x. d. CD18, control. Characteristic distribution of positive cells, scattered in the lamina propria $\left(^{*}\right)$ and concentrated below the crypt epithelium (arrows). 10x. e. Ki-67, adenocarcinoma. Numerous cells have strong, nuclear staining (arrows). 40x. f. Ki67, adenoma. Numerous cells have strong, nuclear staining (arrows). 40x. g. $\beta$-catenin, adenocarcinoma. Strong staining of neoplastic epithelial cells. Both cytoplasmic (arrows) and nuclear (arrowheads) staining are evident. 40x. $\mathbf{h}$. $\beta$-catenin, crypt epithelium from control. Moderate granular staining of cytoplasm only. The signal seems to partially follow the contour of the cell membrane (arrows). 40x 
Table $2 \mathrm{IHC}$ score of $\beta$-catenin in tumors and controls (median, IQR and p-value)

\begin{tabular}{llll}
\hline & $\begin{array}{l}\boldsymbol{P} \text {-value } \\
\boldsymbol{\beta} \text {-catenin } \\
\text { cytoplasma }{ }^{1}\end{array}$ & $\begin{array}{l}\boldsymbol{P} \text {-value } \\
\boldsymbol{\beta} \text {-catenin signal strength }\end{array}$ & $\begin{array}{l}\boldsymbol{P} \text {-value } \\
\boldsymbol{\beta} \text {-catenin distribution }\end{array}$ \\
\hline Adenoma vs controls & $P=0.044$ & $P<0.0001$ & $P=0.03$ \\
& $2.25(1.5-3)$ vs & $2.5(2-3)$ vs & $1(1-1.5)$ vs \\
& $1(1-1.75)$ & $0(0-0)$ & $0(0-0)$ \\
Adenocarcinoma vs controls & $P=0.002$ & $P=0.02$ & $P=0.02$ \\
& $2(2-3)$ vs & $0.25(0-1.88)$ vs & $0.25(0-1)$ vs \\
Adenoma vs adenocarcinomas & $1(1-1.75)$ & $0(0-0)$ & $0(0-0)$ \\
& $P>0.5$ & $P=0.005$ & $P=0.03$ \\
& $2.25(1.5-3)$ vs & $2.5(2-3)$ vs & $1(1-1.5)$ vs \\
\hline
\end{tabular}

${ }^{1}$ Adenocarcinoma $(n=12)$, Adenoma $(n=18)$, Controls $(n=8)$

2 Adenocarcinoma $(n=12)$, Adenoma $(n=16)$, Controls $(n=9)$

${ }^{3}$ Adenocarcinoma $(n=12)$, Adenoma $(n=16)$, Controls $(n=9)$

moderate or strong staining of the nucleus. The presence of nuclear $\beta$-catenin expression (signal strength and distribution) was significantly higher in adenomas compared to adenocarcinomas (Kruskal-Wallis test, $p<$ 0.05, Table 2).

Results of the IHC-scores can be found in Additional file 1.

\section{Discussion}

Colorectal carcinoma develops from clonal expansion of genetically altered cells in humans [9] and dogs [10-14]. Although these genetic changes in tumors are well described, there are also other lesser-known mechanisms involved. Specifically, infiltrative inflammatory cells contribute to tumor progression by promoting cancer growth by releasing proangiogenic or pro-metastatic mediators or suppressing growth through an anti-tumor immune response [29, 30].

To the best of our knowledge, this is the first study focusing on inflammation in canine colorectal carcinogenesis. We found fewer epithelial CD3+ cells, representing $\mathrm{T}$-cells, in colorectal adenomas and adenocarcinomas compared to control samples. Moreover, in adenomas, expression levels of CD18, representing histiocytes, were lower than controls.

Previous studies in humans with CRC have found that high T-cell proliferation is advantageous and associated with increased survival [23, 24, 31]. This association is believed to be due to T-cells initiating a cell-mediated immune response against target antigens on cancerous cells [32]. The lower tumor T-cell infiltration in our work may indicate the opposite; a reduced immune defense against tumor progression. Another explanation for the lower number of TIIs may be a lower expression of tumor-associated antigens by cancerous cells resulting in an inadequate anti-tumor immune response, and subsequently in a lower number of T-cells migrating to the tumor site [33]. The role of T-cells in canine carcinogenesis is not well described. In dogs with nasal carcinomas,
CD3+ cells were reduced compared to normal nasal tissue [34]. In dogs with mammary cancer, high infiltrations of T-cells were associated with a poorer prognosis [35] and another study of dogs with brain gliomas, found that $\mathrm{T}$-cell infiltrations were higher in high-grade glioma vs low-grade glioma [33, 36]. Importantly, CD3+ cells are indiscriminate markers for $\mathrm{T}$-cells and do not differentiate between different subpopulations, with potentially opposing functions [37, 38]. For example, CD8+ and CD4+ T-cells have cytotoxic and immunomodulating properties, respectively [23]. Another marker, FoxP3, represents T-regulatory (Treg) cells, the presence of which may inhibit an efficient immune defense against tumor development [39]. Studies in humans with colorectal cancer have found an association between a low CD3+/FoxP3 ratio and shortened survival [40]. Thus, in order to clarify the potential role of T-cells in canine CRC, the subpopulations of $\mathrm{T}$-cells need to be characterized.

In the present study, we found that expression levels of CD18+ cells were lower in adenomas compared to controls, which may indicate a reduced number of tumor-associated macrophages (TAMs) in adenomas $[26,41]$. Studies in dogs have found that high levels of TAMs are associated with metastases and a poor prognosis in dogs with mammary adenocarcinomas [42, 43] and hemangiosarcomas [44]. As the CD18+ cells were increased in adenocarcinomas relative to adenomas, this might imply that TAMs are associated with malignancy. However, since the highest level of CD18 was observed in control tissue in our work, a more complex malignancy pattern may exist, and characterization of markers specific for TAMs is necessary to clarify the role of TAMs in canine CRC.

In humans, inflammation dominated by lymphocytes and plasma cells is more dominant in CRC compared to normal colonic mucosa [45], and non-steroidal antiinflammatory treatment seems to be effective [46]. Despite our results showing a low presence of T-cells and histiocytes in canine colorectal tumors, anti-inflammatory 
treatment is often used and may be effective. Dogs with rectal polyps of varying malignancy, with minor infiltration of inflammatory cells treated with piroxicam rectally, improved with regards to clinical signs and size of tumors $[47,48]$. This indicates that suppressing inflammation in these cases may be advantageous despite a lack of histologically confirmed inflammation. However, the efficacy of treatment with non-steroidal anti-inflammatory drugs may also depend on whether the tumor expresses cyclooxygenase (COX) [49].

Results from our study confirm previous descriptions of increased $\beta$-catenin expression in the cytoplasm and nucleus of cancerous cells compared to healthy colonic cells $[10,16,50]$. One study showed that this protein might correlate with tumor stage [10]. However, in the present study, the magnitude of nuclear $\beta$-catenin staining did not appear to correlate with tumor stage, as the adenoma samples demonstrated stronger nuclear $\beta$ catenin staining than the adenocarcinomas [16]. However, we cannot exclude the possibility that subtle changes may not have been detected with light microscopy. Other methods such as electron microscopy may be more sensitive for this purpose. Nevertheless, the lack of consistency among studies may be explained by several factors, including different histopathological methods used for tumor classification [51, 52]. A recent canine study showed that adenocarcinoma may be divided into subtypes based on whether they show polypoid or non-polypoid grow patterns [53]. Moreover, these tumors may also consist of different subtypes based on genetic alterations, as demonstrated in human CRC [54]. However, classification methods based on genetic markers are lacking in dogs. As in humans, adenocarcinoma in dogs could also be divided into subtypes which may benefit the evaluation of inflammatory markers in colorectal carcinogenesis.

The dogs with colorectal adenoma and adenocarcinoma consisted of various breeds, including English Setter and German Shepherd, each represented by three individuals. The latter breed is overrepresented in previous case reports of dogs with colorectal adenoma and adenocarcinoma $[6,8,12,55]$. In the majority of dogs in our study (18/31 dogs), tumors were present in the rectum, and only four dogs had tumors in the colon. Similarly, in a study of 78 dogs with colorectal adenocarcinomas, over $85 \%$ of dogs had rectal tumors [6].

One limitation of this study is the low number of dogs, particularly in the control group. Due to the use of archived samples, clinical data are incomplete; thus, precluding further analyses, including survival analyses. Furthermore, the markers used to characterize inflammation in this study, CD3 and CD18, are insufficient to provide a complete picture of the immunophenotype, including T-cell subpopulation, TAMs and cytokine profiles associated with these cells [56]. Frozen samples are necessary for immunophenotype analyses and were unfortunately not available in the present study. The scoring of IHC signals is also a factor that may have influenced our results. For the Ki67 tumor scoring, we used a semi-quantitative scoring approach based on a subjective assessment of the IHC signals. This approach could have been replaced by more standardized methods such as computerized image analyses, which have been used in other studies scoring Ki67 in canine epithelial tumors $[18,57]$.

Although the control dog group was heterogeneous, the ages represented were comparable to those of the dogs with tumors. Healthy young laboratory dogs are commonly used as controls [16]. As age affects the degree of gastrointestinal inflammation $[58,59]$, it may be more relevant to include older dogs as controls, as in our study.

Markers are needed to distinguish adenomas and adenocarcinomas and thus evaluate prognosis. In humans, inflammatory markers have been suggested as part of the classification of malignant tumors [60]. This methodology, called "immunoscoring" may also be useful to distinguish adenomas from adenocarcinomas in dogs, and future studies should aim to determine the types and magnitudeof immune cell infiltration in canine colorectal tumors.

\section{Conclusions}

In this work, we found a decreased presence of CD3+ cells, representing T-cells, in adenomas and adenocarcinomas compared to controls, as well as reduced presence of CD18+ cells, representing histiocytes, in adenomas compared to controls. The lack of these inflammatory cells may have implications for tumor progression. Future studies are needed to further clarify the role of TIIs in the development of canine colorectal cancer.

\section{Methods}

This retrospective case-control study was performed on archived formalin-fixed paraffin-embedded tissue samples, collected for clinical purposes and submitted to NMBU during the period from 1998 to 2015. Owner consent was obtained for the samples to be used for research.

Inclusion criteria for this study were colorectal tissue from dogs with histologically confirmed colorectal adenoma or adenocarcinoma. Eighteen adenomas and 13 adenocarcinomas were included. Colonic tissue for control purposes was collected at necropsy from dogs euthanized for reasons not involving the gastrointestinal tract $(n=9)$. 


\section{Selection of cases and control dogs}

For each case, information about the breed, gender, age, histopathological diagnosis, tumor localization, sampling technique and treatment were obtained from the clinical record (Table 1). The dogs with colorectal adenomas and adenocarcinomas were of various breeds represented by both genders and had a median age of 8 years [min-max, 2-14]). Colorectal mucosal samples were collected from these dogs during surgery $(n=9)$, colonoscopy $(n=8)$, or necropsy $(n=2)$, and in 12 cases by an unknown procedure (Table 1$)$.

The control dogs that contributed the healthy colonic samples consisted of various breeds from both genders and had a median age of 8 years [3-15] (Table 1).

\section{Tissue samples}

Tissue specimens were fixed in $4 \%$ neutral buffered formalin, processed routinely, embedded in paraffin wax, cut into $4 \mu \mathrm{m}$ thick sections and stained with haematoxylin and eosin (HE).

The histopathological diagnoses were evaluated by a board-certified veterinary pathologist (GG), according to the guidelines for classification of canine colorectal adenomas and adenocarcinomas [52]. These guidelines suggest that tumors are classified as adenocarcinoma only if neoplastic cells invade the muscularis mucosa. Although some of the tumors contained cellular features strongly indicative of malignancy, they were still classified as adenomas if no invasion of the basal lamina was found [52].

\section{Immunohistochemistry}

The following antibodies were used; mouse anti- $\beta$ catenin (BD Biosciences, Franklin Lakes, New Jersey), rabbit-anti-CD3 (DAKO, A 0452 North America Inc., California), mouse anti-dog-CD18 (Leucocyte Antigen Laboratory, California) and anti-Ki67 (Abcam, cat no. ab15580, Cambridge).

The sections were heat treated for antigen retrieval by autoclaving at $121^{\circ} \mathrm{C}$ for $15 \mathrm{~min}$ in $0,01 \mathrm{M}$ citric acid $\mathrm{pH} 6.0$ for CD3 and Ki67, and in the microwave in $\mathrm{pH}$ 6.6 Target Retrieval Solution (DAKO, Glostrup, Denmark) for CD18 and pH 9.1 tris-EDTA buffer for $\beta$ catenin.

Endogenous peroxidase activity was inhibited with blocking reagent for $10 \mathrm{~min}$ (DAKO Envision systemHRP AEC REF K 4009 for CD3 and 3.0\% H2O2 in methanol for Ki67, $\beta$-catenin and CD18). Non-specific antigenic sites were blocked with $5 \%$ bovine serum albumin (BSA) in Tris-buffered saline (TBS) for CD3, $1 \%$ normal goat serum (Vector/Bioteam) in 5\% BSA/TBS for Ki67, 2\% BSA in TBS for $\beta$-catenin and 10\% normal goat serum in PBS for CD18. The sections were incubated at room temperature with the following primary antibodies, dilutions and incubation times: rabbit antiCD3 (1:100 in 2,5\% BSA, $60 \mathrm{~min}$ ), rabbit anti-Ki67 (1: 1000 in 2,5\% BSA/ TBS, $60 \mathrm{~min}$ ), mouse anti-dog-CD18 ( $1: 100$ in $10 \%$ goat serum, $30 \mathrm{~min})$ or mouse anti- $\beta$-catenin (1:2500 in 1\% BSA/TBS, $60 \mathrm{~min})$. Sections were then incubated for $30 \mathrm{~min}$ with secondary antibody from the DAKO Envision-kit for CD3, CD18 and B-catenin, and goat anti-rabbit (DAKO, E 432) diluted 1:50 with $2 \%$ normal goat serum for Ki67. The Ki67- sections were then incubated for $30 \mathrm{~min}$ with Elite -ABC- kit (VECT ASTAIN PK-6100) at diluted 1:50 in TBS. Color was revealed for 10 to $15 \mathrm{~min}$ using DAKO Envision systemHRP AEC for CD3, CD18 and $ß$-catenin, and the substrate solution (IMMPACT AEC PEROXIDASE SUBSTRATE SK-4205) for Ki67. Between the various steps, the sections were rinsed thoroughly in TBS. Finally, the sections were counterstained with haematoxylin solution for $45 \mathrm{~s}$ and mounted. Negative control staining was performed by replacing the primary antibodies with nonimmunized goat serum and showed no staining. Lymph node sections were used as positive control tissue for the CD18 and CD3 staining. A canine skin tumor with numerous mitoses served as a positive control for the Ki67 staining. For $\beta$-catenin, the sample tissue itself served as an internal positive control, as all samples contained normal intestinal epithelium, and the cell membranes of these cells were expected to be positive for $\beta$-catenin.

\section{Evaluation of immunohistochemistry}

The sections were reviewed in a blinded fashion by the pathologists and were analyzed subjectively. Two pathologists evaluated the IHC score individually and agreed on the final score (CD18 and $\beta$-catenin; GG and RR, Ki67 and CD3; GG and ØK). For all sections, the IHC score was determined by evaluating the entire specimen.

For $\beta$-catenin, the scoring scheme included the prevalence of cells with a positively stained nucleus, using the following grading system: 0 : no cells with a positive nuclear staining, $1:<1 / 3$ of cells with a positive nuclear staining, $2: 1 / 3-2 / 3$ of cells with a positive nuclear staining and 3: $>2 / 3$ of cells with a positive nuclear staining. Furthermore, the $\beta$-catenin staining intensity in the cytoplasm and the nucleus was scored from 0 to 3 (no staining, weak staining, moderate staining and strong staining).

An absence of nuclear staining for Ki67 was considered negative for this antigen. Ki67 scoring was only evaluated in the epithelium.

CD3+ cells were defined by clearly stained cytoplasm in the epithelium and within cells in the lamina propria.

For CD3, Ki67 and CD18, a semi-quantitative scoring scheme based on the prevalence of positively stained cells was applied, using the following grading system: 0: no staining, 1: few positive cells, 2: a moderate number 
of positive cells, and 3: many positive cells throughout the examined tissue.

The scores of the two pathologists were averaged, resulting in one score for each variable. If the difference between the two pathologists deviated by more than one grade (8 out of 228 scores), the slides were reviewed and discussed, resulting in a final score.

\section{Statistical analysis}

The difference in demographic factors and the IHC score between dogs with adenoma, adenocarcinoma and control dogs were analysed using non-parametric tests (Wilcoxon test and Kruskal-Wallis test) JMP 14 (SAS, USA). A $P$-value $<0.05$ was considered significant for all statistical tests. The median values and interquartile ranges (IQR, 25-75\%) are listed. As the staining for all the antigens was not successful in all samples, the number of samples included is stated for each statistical test.

\section{Abbreviations}

CRC: Colorectal cancer; Tlls: Tumor-infiltrating immune cells; CD: Cluster of differentiation; TAMs: Tumor-associated macrophages; COX: Cyclooxygenase

\section{Supplementary Information}

The online version contains supplementary material available at https://doi. org/10.1186/s12917-021-02829-6.

Additional file 1. $\mid \mathrm{HC}$ score of $\mathrm{CD} 3, \mathrm{CD} 18, \mathrm{Ki} 67$ and $\beta$-catenin in tumorand control samples.

\section{Acknowledgements}

A poster of this study was presented at the 30th ECVIM-CA online congress 2-5 september 2020 (https://onlinelibrary.wiley.com/doi/full/10.1111/jvim.15924). The authors would like to thank the veterinary students Iselin Lyngholm Klinkenberg and Jorunn Karina Skadsem Gil, who contributed with the preparation of samples for immunohistochemistry analyses and Hannah Harjen for the English language review.

\section{Authors' contributions}

$\mathrm{KH}, \mathrm{ES}$ and GG designed the study. LT and RR performed laboratory work. $\varnothing K, G G$ and RR performed the IHC scoring. KH performed statistical analysis. $\mathrm{KH}$ wrote the manuscript with contributions from all authors during manuscript preparation. All authors read and approved the final manuscript.

\section{Funding}

The Norwegian Research Foundation for Canine Cancer provided financial support. This funding source had no role in study design, data collection and analyses, decision to publish, or preparation of the manuscript.

\section{Availability of data and materials}

The datasets used and/or analyzed during the current study are available from the corresponding author on reasonable request.

\section{Declarations}

\section{Ethics approval and consent to participate}

Dog-owners gave consent for using samples for research in cancer diseases. These samples had been taken for clinical purposes, and samples were archived and used for this study. This study used archived samples and ethical approval was deemed unnecessary according to Norwegian Food Safety Authority and national regulations (LOV-2009-06-19-97/FOR-1996-0115-23).
Consent for publication

Not applicable.

\section{Competing interests}

The authors declare no conflict of interest.

\section{Author details}

${ }^{1}$ Department of Companion Animal Clinical Sciences, Faculty of Veterinary Medicine, Norwegian University of Life Sciences (NMBU), Oslo, Norway. ${ }^{2}$ Department of Preclinical Sciences and Pathology, Faculty of Veterinary Medicine, Norwegian University of Life Sciences (NMBU), Oslo, Norway.

${ }^{3}$ Department of Animal Health, Norwegian Veterinary Institute, Section for Biohazard and Pathology, Oslo, Norway.

Received: 23 July 2020 Accepted: 5 March 2021

Published online: 12 March 2021

\section{References}

1. Ferlay J, Ervik M, Lam F, Colombet M, Mery L, Piñeros M et al. Global Cancer Observatory: Cancer Today. Lyon, France: International Agency for Research on Cancer. Available from: https://gco.iarc.fr/today, Accessed 5 Dec 2018.

2. Lingeman $\mathrm{CH}$, Garner FM. Comparative study of intestinal adenocarcinomas of animals and man. J Natl Cancer Inst. 1972;48(2):325-46.

3. Johnson RL, Fleet JC. Animal models of colorectal cancer. Cancer Metastasis Rev. 2013;32(1-2):39-61.

4. Ford AC, Van Zanten SV, Rodgers CC, Talley NJ, Vakil NB, Moayyedi P. diagnostic utility of alarm features for colorectal cancer: systematic review and meta-analysis. Gut 2008;57(11):1545-1553.

5. Assersohn L, Norman A, Cunningham D, Benepal T, Ross PJ, Oates J. Influence of metastatic site as an additional predictor for response and outcome in advanced colorectal carcinoma. Br J Cancer. 1999;79(11-12): 1800-5.

6. Church EM, Mehlhaff CJ, Patnaik AK. Colorectal adenocarcinoma in dogs: 78 cases (1973-1984). J Am Vet Med Assoc. 1987;191(6):727-30.

7. Patnaik AK, Hurvitz Al, Johnson GF. Canine intestinal adenocarcinoma and carcinoid. Vet Pathol. 1980;17(2):149-63.

8. Schäffer E. Incidence and types of canine rectal carcinomas. J Small Anim Pract. 1968;9(10):491-6.

9. Vogelstein B, Fearon ER, Hamilton SR, Kern SE, Preisinger AC, Leppert M, et al. Genetic alterations during colorectal-tumor development. N Engl J Med. 1988;319(9):525-32.

10. McEntee MF, Brenneman KA. Dysregulation of beta-catenin is common in canine sporadic colorectal tumors. Vet Pathol. 1999:36(3):228-36.

11. Youmans L, Taylor C, Shin E, Harrell A, Ellis AE, Seguin B, et al. Frequent alteration of the tumor suppressor gene APC in sporadic canine colorectal tumors. PLoS One. 2012;7(12):e50813.

12. Valerius KD, Powers BE, McPherron MA, Hutchison JM, Mann FA, Withrow SJ. Adenomatous polyps and carcinoma in situ of the canine colon and rectum: 34 cases (1982-1994). J Am Anim Hosp Assoc. 1997;33:156-60.

13. Tang J, Le S, Sun L, Yan X, Zhang M, Macleod J, et al. Copy number abnormalities in sporadic canine colorectal cancers. Genome Res. 2010;20(3): 341-50.

14. Tang J, Li Y, Lyon K, Camps J, Dalton S, Ried T, et al. Cancer driverpassenger distinction via sporadic human and dog cancer comparison: a proof-of-principle study with colorectal cancer. Oncogene. 2014;33(7):81422.

15. Boman BM, Fields JZ. An APC:WNT counter-current-like mechanism regulates cell division along the human colonic crypt Axis: a mechanism that explains how APC mutations induce proliferative abnormalities that drive Colon Cancer development. Front Oncol. 2013;3:244.

16. Aresu L, Pregel P, Zanetti R, Caliari D, Biolatti B, Castagnaro M. E-cadherin and beta-catenin expression in canine colorectal adenocarcinoma. Res Vet Sci. 2010;89(3):409-14.

17. Saito T, Chambers JK, Nakashima K, Uchida E, Ohno K, Tsujimoto H, et al. Histopathologic features of colorectal adenoma and adenocarcinoma developing within inflammatory polyps in miniature dachshunds. Vet Pathol. 2018;55(5):654-62.

18. Woldemeskel M, Hawkins I, Whittington L. Ki-67 protein expression and tumor associated inflammatory cells (macrophages and mast cells) in canine colorectal carcinoma. BMC Vet Res. 2017;13(1):111. 
19. Scholzen T, Gerdes J. The Ki-67 protein: from the known and the unknown. J Cell Physiol. 2000;182(3):311-22.

20. Cui G, Goll R, Olsen T, Steigen SE, Husebekk A, Vonen B, et al. Reduced expression of microenvironmental Th1 cytokines accompanies adenomascarcinomas sequence of colorectum. Cancer Immunol Immunother. 2007; 56(7):985-95.

21. Cui G, Shi Y, Cui J, Tang F, Florholmen J. Immune microenvironmental shift along human colorectal adenoma-carcinoma sequence: is it relevant to tumor development, biomarkers and biotherapeutic targets? Scand J Gastroenterol. 2012;47(4):367-77.

22. Francescone R, Hou V, Grivennikov SI. Cytokines, IBD, and colitis-associated cancer. Inflamm Bowel Dis. 2015;21(2):409-18.

23. Galon J, Costes A, Sanchez-Cabo F, Kirilovsky A, Mlecnik B, Lagorce-Pages C, et al. Type, density, and location of immune cells within human colorectal tumors predict clinical outcome. Science. 2006;313(5795):1960-4.

24. Dahlin AM, Henriksson ML, Van Guelpen B, Stenling R, Oberg A, Rutegard J, et al. Colorectal cancer prognosis depends on T-cell infiltration and molecular characteristics of the tumor. Mod Pathol. 2011;24(5):671-82.

25. Mazzone A, Ricevuti G. Leukocyte CD11/CD18 integrins: biological and clinical relevance. Haematologica. 1995;80(2):161-75.

26. Kang JC, Chen JS, Lee CH, Chang JJ, Shieh YS. Intratumoral macrophage counts correlate with tumor progression in colorectal cancer. J Surg Oncol. 2010;102(3):242-8.

27. Ohmi A, Tsukamoto A, Ohno K, Uchida K, Nishimura R, Fukushima K, et al. A retrospective study of inflammatory colorectal polyps in miniature dachshunds. J Vet Med Sci. 2012;74(1):59-64.

28. Uchida E, Chambers JK, Nakashima K, Saito T, Ohno K, Tsujimoto H, et al. Pathologic features of colorectal inflammatory polyps in miniature dachshunds. Vet Pathol. 2016:53(4):833-9.

29. Coussens LM, Werb Z. Inflammation and cancer. Nature. 2002;420(6917): 860-7.

30. Formica V, Cereda V, Nardecchia A, Tesauro M, Roselli M. Immune reaction and colorectal cancer: friends or foes? World J Gastroenterol. 2014;20(35): 12407-19.

31. Pagès $F$, Berger $A$, Camus $M$, Sanchez-Cabo F, Costes $A$, Molidor $R$, et al. Effector memory T cells, early metastasis, and survival in colorectal cancer. N Engl J Med. 2005;353(25):2654-66.

32. Boon T, Cerottini JC, Van den Eynde B, van der Bruggen P, Van Pel A. Tumor antigens recognized by T lymphocytes. Annu Rev Immunol. 1994;12:337-65.

33. Pitcovski J, Shahar E, Aizenshtein E, Gorodetsky R. Melanoma antigens and related immunological markers. Crit Rev Oncol Hematol. 2017;115:36-49.

34. Vanherberghen M, Day MJ, Delvaux F, Gabriel A, Clercx C, Peeters D. An immunohistochemical study of the inflammatory infiltrate associated with nasal carcinoma in dogs and cats. J Comp Pathol. 2009;141(1):17-26.

35. Franzoni MS, Brandi A, de Oliveira Matos Prado JK, Elias F, Dalmolin F, de Faria Lainetti $P$, et al. Tumor-infiltrating CD4(+) and CD8(+) lymphocytes and macrophages are associated with prognostic factors in triple-negative canine mammary complex type carcinoma. Res Vet Sci. 2019;126:29-36.

36. Pi Castro D, José-López R, Fernández Flores F, Rabanal Prados RM, Mandara MT, Arús C, et al. Expression of FOXP3 in canine Gliomas: Immunohistochemical study of tumor-infiltrating regulatory lymphocytes. J Neuropathol Exp Neurol. 2020;79(2):184-93.

37. Cobbold S, Metcalfe S. Monoclonal antibodies that define canine homologues of human CD antigens: summary of the first international canine leukocyte antigen workshop (CLAW). Tissue Antigens. 1994;43(3): 137-54.

38. Elwood CM, Hamblin AS, Batt RM. Quantitative and qualitative immunohistochemistry of T cell subsets and MHC class $\|$ expression in the canine small intestine. Vet Immunol Immunopathol. 1997;58(3-4):195-207.

39. Clarke SL, Betts GJ, Plant A, Wright KL, El-Shanawany TM, Harrop R, et al. CD4+CD25+FOXP3+ regulatory T cells suppress anti-tumor immune responses in patients with colorectal cancer. PLoS One. 2006;1:e129.

40. Sinicrope FA, Rego RL, Ansell SM, Knutson KL, Foster NR, Sargent DJ. Intraepithelial effector (CD3+)/regulatory (FoxP3+) T-cell ratio predicts a clinical outcome of human colon carcinoma. Gastroenterology. 2009;137(4): 1270-9.

41. Ruffell B, Affara NI, Coussens LM. Differential macrophage programming in the tumor microenvironment. Trends Immunol. 2012;33(3):119-26.

42. Król M, Pawłowski KM, Majchrzak K, Dolka I, Abramowicz A, Szyszko K, et al. Density of tumor-associated macrophages (TAMs) and expression of their growth factor receptor MCSF-R and CD14 in canine mammary adenocarcinomas of various grade of malignancy and metastasis. Pol J Vet Sci. 2011;14(1):3-10.

43. Raposo T, Gregório H, Pires I, Prada J, Queiroga FL. Prognostic value of tumour-associated macrophages in canine mammary tumours. Vet Comp Oncol. 2014;12(1):10-9.

44. Regan DP, Escaffi A, Coy J, Kurihara J, Dow SW. Role of monocyte recruitment in hemangiosarcoma metastasis in dogs. Vet Comp Oncol. 2017;15(4):1309-22.

45. Banner BF, Savas L, Baker S, Woda BA. Characterization of the inflammatory cell populations in normal colon and colonic carcinomas. Virchows Arch B Cell Pathol Incl Mol Pathol. 1993;64(4):213-20.

46. Coyle C, Cafferty FH, Langley RE. Aspirin and colorectal Cancer prevention and treatment: is it for everyone? Curr Colorectal Cancer Rep. 2016;12:2734.

47. Knottenbelt CM, Simpson JW, Chandler ML. Neutrophilic leucocytosis in a dog with a rectal tumour. J Small Anim Pract. 2000;41(10):457-60.

48. Knottenbelt CM, Simpson JW, Tasker S, Ridyard AE, Chandler ML, Jamieson PM, et al. Preliminary clinical observations on the use of piroxicam in the management of rectal tubulopapillary polyps. J Small Anim Pract. 2000; 41(9):393-7.

49. Knottenbelt C, Mellor D, Nixon C, Thompson H, Argyle DJ. Cohort study of COX-1 and COX-2 expression in canine rectal and bladder tumours. J Small Anim Pract. 2006;47(4):196-200.

50. Restucci B, Martano M. G DEV, Lo Muzio L, Maiolino P. expression of Ecadherin, beta-catenin and APC protein in canine colorectal tumours. Anticancer Res. 2009;29(8):2919-25.

51. Head KW. Histological classification of tumors of the alimentary system of domestic animals: published by the armed forces Institute of Pathology in cooperation with the American registry of pathology and the World Health Organization collaborating Center for Worldwide Reference on comparative oncology; 2003.

52. Munday JS, Löhr CV, Kiupel M. Tumors of the alimentary tract in: tumors in domestic animals. Fifth ed. North Carolina, US: Wiley Blackwell; 2016. p. 499601.

53. Saito T, Nibe K, Chambers JK, Uneyama M, Nakashima K, Ohno K, et al. A histopathological study on spontaneous gastrointestinal epithelial tumors in dogs. J Toxicol Pathol. 2020;33(2):105-13.

54. Kudryavtseva AV, Lipatova AV, Zaretsky AR, Moskalev AA, Fedorova MS, Rasskazova AS, et al. Important molecular genetic markers of colorectal cancer. Oncotarget. 2016;7(33):53959-83.

55. Holt PE, Lucke VM. Rectal neoplasia in the dog: a clinicopathological review of 31 cases. Vet Rec. 1985;116:400-5.

56. West NR, McCuaig S, Franchini F, Powrie F. Emerging cytokine networks in colorectal cancer. Nat Rev Immunol. 2015;15(10):615-29.

57. Pereira RS, Schweigert A, Dias de Melo G, Fernandes FV, Sueiro FA, Machado GF. Ki-67 labeling in canine perianal glands neoplasms: a novel approach for immunohistological diagnostic and prognostic. BMC Vet Res. 2013;9:83.

58. Day MJ. Ageing, immunosenescence and inflammageing in the dog and cat. J Comp Pathol. 2010;142(Suppl 1):S60-9.

59. Kleinschmidt S, Meneses F, Nolte I, Hewicker-Trautwein M. Characterization of mast cell numbers and subtypes in biopsies from the gastrointestinal tract of dogs with lymphocytic-plasmacytic or eosinophilic gastroenterocolitis. Vet Immunol Immunopathol. 2007;120(3-4):80-92.

60. Galon J, Mlecnik B, Bindea G, Angell HK, Berger A, Lagorce C, et al. Towards the introduction of the 'Immunoscore' in the classification of malignant tumours. J Pathol. 2014;232(2):199-209.

\section{Publisher's Note}

Springer Nature remains neutral with regard to jurisdictional claims in published maps and institutional affiliations. 